\title{
Transforming anatomy education: then and now
}

\author{
Anjali Singal ${ }^{1}$ (i) \\ Received: 23 November 2021 / Accepted: 12 December 2021 / Published online: 17 January 2022 \\ (c) The Author(s), under exclusive licence to Japanese Association of Anatomists 2021
}

Evolution is an enduring process and the one who manages to change "survives". Anatomy, too, has transformed in every aspect—from procurement, embalming and preservation of cadavers to curriculum, books, teaching methods and assessments. Factors such as the increasing number of medical students, limited resources, and lesser time devoted to the subject per the new curriculum have compelled every anatomist to unravel new techniques. Anatomy has been transforming for the last two decades, but the COVID-19 pandemic has revolutionized anatomy education. "Dissection should be part of medical education curriculum or not" was the hot topic of discussion among the anatomy fraternity, and the pandemic provided a forced chance to explore "cadaverless" anatomy education scenario worldwide. During this crisis, anatomists shifted to the virtual platform and explored innovative technology, pedagogical approaches, and assessment methods with the least delays.

\section{Dissection: then and now}

The cadaveric dissection converged through various phases-from stealing corpses to permitted and legal use of cadaver and now modern high-tech, almost cadaverless anatomy. Even though e-learning packages in the market claim to deliver three-dimensional anatomy, every anatomist knows what feeling one gets when their scalpels cut through the heaven-aboded silent teachers. Though this millennial generation of "active" learners appreciates new technology, group learning, and active teaching methods, some students may not be technology friendly and face difficulty. Traditional anatomy teaching includes dissection and formal lectures. Though passive, but formal lectures are still the pillar of anatomy education, they allow conveying

Anjali Singal

anjali_singal@rediffmail.com

1 Department of Anatomy, All India Institute of Medical Sciences, Bathinda 151001, India a large amount of information in an organized manner to a large group of students in less time. Modern lectures are not limited to chalk and board, but various techniques, such as interactive whiteboards, animated videos, three-dimensional images, iconic hand gestures, and others, make lectures more engaging.

\section{From simple microscope to screen}

Traditional microscopy is shifting to virtual microscope software, i.e., observing virtual slides with a virtual microscope on a digital device. Students will be found gazing at the screen and not genuinely learning how to manually focus the microscope on seeing those stained slides and learning the real deal. We should not replace the microscope with virtual means; instead, we may implicate screen methods for lecture or revision purposes.

\section{Embryology/radiology/surface anatomy evolution}

Embryology is an integral part of anatomy. However, students usually find it tough to grasp the concepts of simultaneous changes and imagine a developing embryo's spatial orientation. Inclusion of animations, focus on clinical application, learning through case scenarios, ultrasound, and autopsy images from embryos and fetuses in modern embryology classes may improve students' interest and understanding of the subject. With innovation in imaging techniques, radiology classes advanced from X-rays to CT scans, MRIs, and the latest techniques. Digital images are taking over hard copies of X-rays during education. Traditional surface anatomy provides an opportunity to practice physical examination of different landmarks on cadavers and prepares the students for clinical training. Such traditional anatomy practice is the best even in modern anatomy teaching. 


\section{Anatomy assessment}

Assessment is a significant milestone in medical education. The change in curriculum gradually changed the assessment method too. The pattern of the theory paper evolved from long answer questions to the single best option, one-word answer, and the long structured questions to assess knowledge and decrease bias in relative scores of students.

The anatomy education system cannot be universal, as each institute/state/country may vary in finances, procurement of cadavers, faculty-student ratio, or other resources. The goal, however, is the same, i.e., to prepare better health professionals. Every anatomy faculty has to select the most suitable way according to their setup and endow it with accessible novel technologies. Anatomists should try their best, to look for the possibilities of cadaveric dissection for a more profound knowledge of anatomy as we can agree "cadaver is the best learning source next to living humans." However, living anatomy teaching is in trend at some setups but has its limitations.

\section{Guidelines for future anatomy lectures and dissection}

The anatomy content and information have increased and the assigned hours for subject teaching decreased; this factor may emphasize the use of time saving and effective pedagogical tools. It is a well proven fact that there is no single pedagogical method or model in anatomy education, documented to be superior to another (Johnson et al. 2012; Wilson et al. 2018). Some suggested strategies are:

1. The latest technology may significantly be used to learn the complex concepts in the subject and to supplement passive lectures.

2. Active learning methods such as problem-based learning, computer-assisted learning, and case studies may be incorporated in the curriculum (Papa and Vaccarezza 2013).

3. Cadaveric dissection is a great learning tool in anatomy, but needs constant resources, great infrastructure and time. Keeping in view the above issues, prosections and plastinated specimens should be the second choice as they are real human specimens, followed by threedimensional interactive dissection tables which provide the student with the opportunity of exploring life-size anatomy, and other learning modalities (Drake et al. 2009; Suárez-Escudero et al. 2020).

4. If limited resources for cadaveric dissection are available, anatomy and surgery postgraduate students or residents may be given preference to use these materials.

One must remember that the scope for designing new educational tools to deliver the best anatomy education is ever expanding and unlimited. Now is the time to complement conventional anatomical teaching with innovation, which demands modern anatomists to combine traditional and technology-savvy scientists.

Funding Nil.

\section{References}

Drake RL, McBride JM, Lachman N, Pawlina W (2009) Medical education in the anatomical sciences: the winds of change continue to blow. Anat Sci Educ 2:253-259

Johnson EO, Charchanti AV, Troupis TG (2012) Modernization of an anatomy class: from conceptualization to implementation. A case for integrated multimodal multidisciplinary teaching. Anat Sci Educ 5:354-366

Papa V, Vaccarezza M (2013) Teaching anatomy in the XXI century: new aspects and pitfalls. Sci World J. 1-5. https://goo.gl/2pL4B5

Suárez-Escudero JC, Posada-Jurado MC, Bedoya-Muñoz LJ, UrbinaSánchez AJ, Ferreira-Morales JL, Bohórquez-Gutiérrez CA (2020) Teaching and learning anatomy pedagogical methods, history, the present and tendencies. Acta Med Colomb. https:// doi.org/10.36104/amc. 2020.1898

Wilson AB, Miller CH, Klein BA, Taylor MA, Goodwin M, Boyle EK et al (2018) A meta-analysis of anatomy laboratory pedagogies. Clin Anat 31:122-133

Publisher's Note Springer Nature remains neutral with regard to jurisdictional claims in published maps and institutional affiliations. 